CAUSATION

\title{
Childhood acute lymphoblastic leukaemia was not linked to residential exposure to power line magnetic fields
}

\author{
Linet MS, Hatch EE, Kleinerman RA, et al. Residential exposure to magnetic fields and acute lymphoblastic leukemia in children. $N$ \\ Engl J Med 1997 Jul 3;337:1-7.
}

\section{Objective}

To determine whether an association exists between residential exposure to magnetic fields from power lines and the development of childhood acute lymphoblastic leukaemia (ALL).

\section{Design}

Case control study.

\section{Setting}

Community based study in 9 states of the USA.

\section{Participants}

Case participants were children who were diagnosed with ALL before the age of 15 years between 1989 and 1994. Control group children were matched for age, race, and the first 8 digits of their telephone number. Magnetic field measurements and residential history were available for 629 children with ALL and 619 control group children who had participated in an initial telephone survey; 463 case control pairs were included in the matched analysis.

\section{Assessment of risk factors}

For children $<5$ years of age, magnetic fields were measured in homes that the children had lived in for $\geqslant 6$ months and spent $\geqslant 70 \%$ of their lives. For children $\geqslant 5$ years of age, measurements were done in the homes in which the children had spent $\geqslant 70 \%$ of the 5 years preceding the diagnosis date. Technicians, who were blinded to the case and control status of the participants, measured magnetic fields for a 24 hour period in each child's bedroom, and for 30 seconds in other rooms and outside the front door. The residential wire code categories were assessed for a subgroup of 416 pairs of participants with stable residences and for the residences of 230 pairs where families had lived during the mother's pregnancy with the participating child.

\section{Main outcome measure}

Risk of ALL according to time weighted average residential magnetic field levels (4 categories of increasing exposure selected a priori) and 2 classifications of wire codes.

\begin{abstract}
Main results
Risk estimates were adjusted for the child's age at diagnosis of ALL, sex, mother's educational level, and family income. Risk of childhood ALL was not associated with magnetic field exposure. At the highest magnetic levels of $\geqslant 0.200 \mu \mathrm{T}$, the odds ratio (OR) for the unmatched analysis was 1.24 (95\% CI 0.86 to 1.79$)$ and for the matched analysis was 1.53 (CI 0.91 to 2.56). The risk did not increase with increasing exposure ( $\mathrm{p}$ for trend $=0.22$ ). Adjustment for family income, parental education level or occupation, birth order, or other residential characteristics did not alter the relation. No association existed between risk of ALL and the highest wire code category for either the Wertheimer-Leeper classification (OR 0.88 , CI 0.48 to 1.63 ) or the Kaune-Savitz classification (OR 1.04, CI 0.65 to 1.66). Risk of ALL was not associated with magnetic field levels or wire codes in the homes that mothers lived in when pregnant with the participants.
\end{abstract}

\section{Conclusion}

No association existed between risk of childhood acute lymphoblastic leukaemia and residential exposure to magnetic fields from power lines.

Sources of funding: in part, National Cancer Institute and the University of Minnesota Children's Cancer Research Fund.

For article reprint: $\operatorname{Dr} M S$ Linet, Division of Cancer Epidemiology and Genetics, National Cancer Institute, Executive Plaza North, Suite 408, Bethesda, MD 20892-7362, USA. Fax +1 3014020207.

Adapted from an abstract published in Evidence-Based Medicine 1998 Mar-Apr;3:43.

\section{Commentary}

The research linking magnetic fields produced by power lines and cancer is controversial because of inconsistent study results. Confidence in the results is often limited by methodological weaknesses and difficulties in measuring exposure to magnetic fields. The study by Linet et al provides the strongest evidence to date that no association exists between magnetic fields and risk of developing ALL.

The strengths of the study are the large sample size, the estimation of cumulative exposure to magnetic fields close to the time of diagnosis, and the blinding of data collectors to the case or control status of the study participants. Of importance is the estimation of prenatal exposure to magnetic fields for mothers of children $<5$ years of age. No risk of ALL and prenatal exposure was found. Although the study data suggest a trend toward increased risk of ALL in the highest wire code categories, the small number of participants with these high levels of exposure make it difficult to draw conclusions.

Acute lymphoblastic leukaemia is the most common form of childhood cancer. The results of this study are relevant to nurse practitioners and paediatric, oncology, and public health nurses working with expectant mothers, infants, and children. Questions that nurses are commonly asked include: my home is situated near power lines; should I move? Or, did the power lines close to my home cause my child's leukaemia? Concerned parents have often spent large amounts of money to reduce electromagnetic fields within the home. The results of this study provide little support for the relation between living in homes with high magnetic field levels or in homes close to electrical transmission or distribution lines and childhood ALL

Denise Bryant-Lukosius, RN, MScN Clinical Nurse Specialist, Haematology/Oncology Hamilton Health Sciences Corporation Hamilton, Ontario, Canada 